\title{
Impacto de los sistemas automatizados en un modelo de consulta de alta resolución
}

\author{
F. ROSA-JIMÉNEZ, L. MARTOS MELGUIZO, A. M. MONTIJANO CABRERA', \\ J. L. ZAMBRANA GARCÍA', M. D. ADARRAGA CANSINO' \\ Líneas de Procesos Generales del Adulto. Hospital "Alto Guadalquivir”. Andújar, Jaén. \\ ${ }^{\text {I} H o s p i t a l ~ d e ~ M o n t i l l a . ~ C o ́ r d o b a ~}$
}

\section{RESUMEN}

Objetivo: Estudiar la eficacia de los sistemas informatizados en las Áreas de Radiología y Laboratorio en la reducción del tiempo de espera de los pacientes en un programa de alta resolución en la consulta externa de medicina interna.

Material y método: Se analizó durante un año la demora de las pruebas diagnósticas solicitadas en el día (laboratorio, radiología simple, ecografía y TAC craneal) y el tiempo de espera de los pacientes según el método convencional (312 peticiones cuyos resultados eran enviados a través de celadores o personal auxiliar) o el método informatizado (457 peticiones vía intranet).

Resultados: Se observó una reducción media del $10 \%$ en la demora global, aunque las diferencias sólo fueron significativas para la TAC $(12,7 \%)$ y el laboratorio (19\%). La reducción media del tiempo de espera del paciente fue del $11,7 \%$ que osciló según fueran una $(7,8 \%)$ o dos $(13,2 \%)$ las pruebas solicitadas. El porcentaje de pacientes que esperaron más de 3 horas la llegada de sus resultados se redujo de forma significativa.

Discusión: El uso de sistemas automatizados de transmisión de las exploraciones complementarias produjo una reducción del tiempo de espera de los pacientes. Sin embargo, los resultados no afectaron de forma homogénea a las distintas técnicas.

PALABRAS CLAVE: Intranet. Demora. Consulta única. Pruebas diagnósticas. Consultas ambulatorias. Alta resolución.
IMPACT OF AUTOMATISED SYSTEMS IN A HIGH RESOLUTION MODEL OF OUTPATIENTS CLINICS

\section{ABSTRACT}

Objective: To analyse the impact of computerised systems in Radiology and Laboratory Departments on the reduction in the waiting time of the patients attending to an Internal Medicine outpatient consultation organised in a high resolution model.

Methods: For one year, we studied the delay in diagnostic explorations (laboratory test, simple radiology, ultrasonic diagnostics and brain scanner) that were all applied for and performed in a single day. We compared the traditional organization ( $n=312$ explorations), in which the results were handed in by hospital auxiliary staff, to a computerised method ( $n=457$ explorations), which was implemented via our intranet.

Results: The global delay decreased $10 \%$ in average, the differences being significant only for the variables "brain scanner" (12.7\%) and "laboratory" (19\%). The average reduction in the waiting time per patient was $11.7 \%$, ranging from $7.8 \%$ (when only one exploration was performed) to $13.2 \%$ (when 2 explorations were carried out). The percentage of patients who needed to wait more than 3 hours to receive their results also diminished significantly.

Conclusion: The implementation of computerised systems reduced the waiting time to receive the results of complementary explorations. However, our results were not homogeneous for the different explorations.

KEY WORDS: Intranet. Delay. Just-one-day consultation. Diagnostic explorations. Outpatient. High resolution.

Rosa-Jiménez F, Martos Melguizo L, Montijano Cabrera AM, Zambrana García JL, Adarraga Cansino MD. Impacto de los sistemas automatizados en un modelo de consulta de alta resolución. An Med Interna (Madrid) 2005; 22: 511-514.

\section{INTRODUCCIÓN}

La problemática médica y social derivada de la existencia de las listas de espera en la asistencia sanitaria pública es una cuestión que gravita sobre los distintos tipos de gestión sanitaria (1). Su importancia es tal que recientemente ha promovido la promulgación de leyes que intentan acotar los tiempos máximos de espera en unos intervalos que oscilen entre lo deseable y lo razonable. Aún no se ha encontrado un modelo ideal que sea aplicable a todos los ámbitos y niveles sanitarios, aunque no se cesa en el desarrollo de nuevas ideas que, al menos, mejoren los modelos tradicionales (2-6). En esta línea, desde su apertura en 1999 nuestro hospital utiliza como modelo asistencial el concepto de consulta única o consulta de alta resolución (CAR) (7) que pretende incluir (en un mismo acto asistencial) la realización de las técnicas diagnósticas necesa- 
rias para llegar a un diagnóstico y su tratamiento. Ello implica la reducción del número de visitas del paciente al hospital evitando la demora de cada prueba solicitada y la consecuente citación posterior a la consulta.

Los rápidos avances obtenidos en el campo de las telecomunicaciones han permitido la implantación de nuevas tecnologías para el tratamiento de la información médica. En algunos casos, los potenciales beneficios en términos de seguridad y eficacia quedan aún por demostrar (8). Recientemente nuestro hospital ha puesto en marcha un sistema de apoyo telemático en las consultas externas lo que permite recibir online los resultados de las pruebas diagnósticas habituales de radiología y laboratorio. Nuestro objetivo en el presente estudio es analizar si el uso de dichos sistemas informatizados redunda en un menor tiempo de espera del paciente en un programa de CAR.

\section{MATERIAL Y MÉTODO}

Nuestro hospital atiende a una población aproximada de 65.000 habitantes. En el programa de CAR el paciente que acude como primera visita a la consulta externa de Medicina Interna es valorado por un facultativo que realiza una historia clínica y su exploración física correspondiente. En función del motivo de consulta se valora si el paciente precisa de la realización de alguna exploración complementaria incluida en la cartera de servicios de dicho sistema (Tabla I). Una vez realizadas las técnicas solicitadas el paciente vuelve a la consulta en el momento en que los resultados estén disponibles. Nuestro hospital dispone de una intranet a la que se accede, a través de un sistema de hardware convencional, desde todas las consultas externas. Los resultados de las exploraciones complementarias son accesibles a través de un software comercial (Pathspeed $\mathrm{Web}^{\circledast}$ para imágenes e informes radiológicos y Weblab ${ }^{\circledast}$ para resultados analíticos).

Durante un año (noviembre-2002 hasta octubre-2003) se recogió el tiempo trascurrido desde que al paciente se le entregaba la solicitud de la/las pruebas complementarias hasta su recepción vía intranet. El estudio se realizó en las dos consultas externas de Medicina Interna en pacientes de primera visita. A efectos prácticos los resultados se agruparon en: pruebas de laboratorio, radiología convencional, ecografía y tomografía axial computerizada (TAC). El resto de peticiones (ECG, espirometría, etc.) no se incluyeron en el estudio. Se analizaron: 1) la demora media de cada grupo de pruebas; 2) el tiempo medio de espera de los pacientes; y 3) el porcentaje de pacientes que esperaron más de tres horas la llegada de sus resultados. Para el tiempo de espera de los pacientes se consideró la demora del método diagnóstico (una prueba diagnóstica) o aquélla que más se retrasó (en caso de solicitar dos o más técnicas). Los resultados obtenidos se compararon con un estudio piloto anterior (9) de las mismas características y diseño realizado entre los meses de noviembre-2000 y octubre-2001. En dicho trabajo los resultados de las pruebas complementarias solicitadas en el programa de CAR eran enviados a la consulta de manera convencional: bien mediante celadores o bien a través del personal auxiliar de la consulta.

Para el estudio estadístico se utilizó el programa SPSS $9.0^{\circledR}$ para Windows ${ }^{\oplus}$, utilizando el test de la $\mathrm{T}$ de Student para la comparación de medias independientes y el test de la $\chi^{2}$ para la comparación de proporciones.
TABLA I

PRUEBAS DIAGNÓSTICAS INCLUIDAS EN LA CARTERA DE SERVICIOS EN EL PROGRAMA DE CONSULTA DE ALTA RESOLUCIÓN

Laboratorio

Hemograma, extensión de sangre periférica, reticulocitos

VSG en la primera hora

Grupo sanguíneo, test de Coombs

directo e indirecto

Estudio de coagulación, dímero D, fibrinógeno

Glucosa, sobrecarga oral de glucosa, test de O'Sullivans

Colesterol total-LDL-HDL, triglicéridos

Proteínas totales, albúmina, inmunoglobulinas

LDH, GOT, GPT, GGT, FFAA, bilirrubina total y directa, amilasa

Gasometría arterial

Digoxina

Sodio, potasio, calcio, cloro, fósforo, osmolaridad

Creatinina, urea

Sideremia, transferrina, ferritina, saturación de transferrina

CPK, PCR, factor reumatoide, ácido úrico, ASLO

Orina (sistemático, sedimento, urea, creatinina, sodio, potasio, test de gestación)

Rosa de Bengala

Radiología convencional Serie ósea

Radiografía de tórax antero-posterior y lateral

Radiografía de abdomen simple

Seriada esofagogastroduodenal con contraste

TAC

Ecografía

TAC craneal sin contraste

Ecografía cervical

Ecografía abdominal

Ecografía de mama

Ecografía de partes blandas

Ecografía doppler venosa de extremidades

Otras

Electrocardiograma 12 derivaciones Ecocardiografía transtorácica

Espirometría

Endoscopia oral

\section{RESULTADOS}

\section{PACIENTES}

En el primer estudio (modelo convencional) se solicitaron 343 pruebas complementarias. De ellas no fueron aceptadas $15(4,4 \%)$ y no se recogieron los datos en 16 casos (total pérdidas 31 [9\%]). Las restantes 312 peticiones provenían de 264 pacientes. En el segundo estudio (modelo informatizado) se 
solicitaron 496 pruebas diagnósticas, de las cuales no fueron aceptadas $14(3 \%)$ y se perdieron los datos de 25 (total pérdidas 39 [8\%]). Las 457 peticiones analizadas correspondían a 380 pacientes. No se observaron diferencias estadísticamente significativas al analizar edad, sexo, día de la semana o mes del año respecto a los parámetros estudiados.

\section{TIEMPOS MEDIOS DE DEMORA DE LAS PRUEBAS COMPLEMENTARIAS}

El análisis de cada una de las pruebas solicitadas evidenció un descenso significativo en el modelo informatizado en los tiempos medios de demora de la TAC (12,7\%; 19,6 minutos)) y de los resultados del laboratorio (19\%; 32,1 minutos) (Tabla II). Al considerar el global de las pruebas solicitadas también se observó una reducción media del 10\% del tiempo de demora (15,3 minutos) con el uso de la intranet respecto al sistema convencional. Los incrementos observados en la ecografía y el decremento en la radiología convencional no alcanzaron significación estadística. tas que acuden a nuestras consultas externas. En unos casos las técnicas diagnósticas no son necesarias. En otros dichas pruebas son aportadas por el paciente (atención primaria, mutuas, etc.) o están en su historia clínica (interconsultas, informes de alta, visitas a urgencias, etc.). En tercer lugar, y como es común con el resto del sistema sanitario, la realización de determinadas pruebas diagnósticas tiene que ser demorada más o menos tiempo (estudios radiológicos con contraste, holter 24 horas, estudios serológicos y de autoinmunidad, etc.). En nuestras consultas de Medicina Interna la solicitud de pruebas complementarias en el programa de alta resolución se aplica, aproximadamente, al $15 \%$ de las primeras visitas (10). En otras unidades de nuestro hospital cuya estructura les hace ser más autosuficientes y menos dependientes de técnicas diagnósticas de otros servicios, el programa de CAR también se aplica con satisfactorios resultados (11-14).

La puesta en marcha de los sistemas informáticos en la transmisión de imágenes, informes y resultados analíticos ha demostrado una reducción en el tiempo de espera de nuestros pacientes. No obstante, los resultados no han sido homogéneos. Se ha observado una disminución global, sobre todo a expensas

TABLA II

TIEMPOS MEDIOS DE DEMORA DE LAS EXPLORACIONES COMPLEMENTARIAS

\begin{tabular}{lcccccc}
\hline Prueba & $n$ & Modelo convencional & $n$ & Modelo informatizado & Diferencia* $^{*}$ & $p$ \\
\hline Laboratorio & 103 & $168,4(51)[40,265]$ & 197 & $136,3(44)[55,280]$ & $-32,1(6)$ & $<0,001$ \\
Radiología simple & 109 & $133,9(49)[15,250]$ & 125 & $128,5(51)[15,270]$ & $-5,4(7)$ & $n$ ns \\
Ecografía & 30 & $138,4(53)[65,270]$ & 42 & $148,1(58)[40,265]$ & $+9,7(13)$ & $n$ ns \\
TAC & 70 & $153,7(64)[55,315]$ & 93 & $134,1(55)[20,270]$ & $-19,6(9)$ & 0,035 \\
Total & 312 & $150,2(55)[15,315]$ & 457 & $134,8(50)[15,280]$ & $-15,3(4)$ & $<0,001$ \\
\hline
\end{tabular}

Resultados expresados en como m (DS) [rangos] en minutos para T de Student. *Diferencia de medias (Error estándar) en minutos.

\section{TIEMPO MEDIO DE ESPERA DEL PACIENTE}

Se observó un descenso del 7,8\% (11 minutos) en el tiempo de espera del grupo de pacientes a los que se les realizó una única prueba diagnóstica respecto a los valores iniciales del modelo convencional (Tabla III). El grupo de pacientes a los que se les solicitaron dos o más técnicas mejoraron sus resultados en un 13,2\% (25,3 minutos). La reducción del tiempo de espera, considerando el global de los pacientes, fue del 11,7\% (17,5 minutos).

\section{ESPERA SUPERIOR A 3 HORAS}

Con el uso de la intranet se observó un aumento del 10,9\% en el porcentaje de pacientes que esperaron menos de 3 horas la llegada de sus resultados (Tabla IV). Éste osciló entre el $8,5 \%$ en los pacientes con una única prueba y el $24,5 \%$ en los se realizaron dos o más.

\section{DISCUSIÓN}

La implantación de un modelo de CAR no implica la realización de pruebas complementarias a todas las primeras visi- de mejoras en los resultados del laboratorio y de la TAC. No encontramos una explicación razonable de por qué esto no alcanza a la radiología convencional y a la ecografía puesto que la sistemática de trabajo es la misma en toda el Área de Radiología y ésta no ha cambiado en los dos periodos analizados.

Por desgracia este modelo es fácilmente saturable. El laboratorio (Área de Biotecnología) ha automatizado la realización de la mayoría de las determinaciones analíticas. Ello permite, dentro del horario previsto (hasta las 12 de la mañana) aceptar (salvo problemas técnicos) cuantas determinaciones se soliciten. Sin embargo, el Área de Radiología debe hacer "malabarismos" entre los pacientes citados ambulatoriamente, las peticiones de los pacientes ingresados, las solicitudes procedentes del área de urgencias y las consultas únicas generadas por las consultas externas de todo el hospital. Los picos asistenciales en urgencias, la ausencia de algún facultativo, el incremento de pacientes ingresados, la estacionalidad de algunas patologías, etc. son algunos de los motivos para que las solicitudes de consulta única puedan no ser aceptadas. A pesar de estos problemas se ha de subrayar que más del $95 \%$ de las peticiones fueron atendidas.

Una encuesta posterior (15) realizada a los profesionales facultativos de nuestro hospital acerca de su opinión sobre la implantación del sistema telemático señaló que cerca del $90 \%$ de los mismos estaban satisfechos con la misma, ya que, amén 
TABLA III

TIEMPO MEDIO DE ESPERA DEL PACIENTE EN FUNCIÓN DEL NÚMERO DE PRUEBAS SOLICITADAS

\begin{tabular}{lcccccc}
\hline Prueba & $n$ & Modelo convencional & $n$ & Modelo informatizado & Diferencia* $^{*}$ & $p$ \\
\hline 1 & 219 & $141,2(57)[15,315]$ & 308 & $130,2(50)[15,280]$ & $-11(5)$ & 0,021 \\
$\geq 2$ & 45 & $191,1(39)[120,265]$ & 72 & $165,8(45)[60,270]$ & $-25,3(9)$ & $<0,001$ \\
Total de pacientes & 264 & $149,7(57)[15,315]$ & 380 & $132,2(51)[15,280]$ & $-17,5(4)$ & $<0,01$ \\
\hline
\end{tabular}

Resultados expresados en como m (DS) [rangos] en minutos para T de Student. *Diferencia de medias (Error estándar) en minutos.

TABLA IV

PORCENTAJE DE PACIENTES CON ESPERA INFERIOR O IGUAL A 3 HORAS

\begin{tabular}{lcccccc}
\hline Pruebas solicitadas & $n$ & Modelo convencional & $n$ & Modelo informatizado & Diferencia* & $p$ \\
\hline 1 & 219 & $169(77,2 \%)$ & 308 & $264(85,7 \%)$ & $8,5 \%$ & 0,013 \\
$\geq 2$ & 45 & $19(42,2 \%)$ & 72 & $48(66,7 \%)$ & $24,5 \%$ & 0,005 \\
Total de pacientes & 264 & $188(71,2 \%)$ & 380 & $312(82,1 \%)$ & $10,9 \%$ & 0,001 \\
\hline
\end{tabular}

de la rapidez presenta otras ventajas (exportación de archivos gráficos, mejora de la calidad de imagen, visualización de exámenes complementarios previos, etc.). En nuestro caso no recogimos información acerca de la satisfacción del usuario sobre este modelo asistencial. Sin embargo, los resultados obtenidos en otras unidades de nuestro hospital a este respecto han sido satisfactorios $(16,17)$.

\section{AGRADECIMIENTOS}

Este trabajo ha sido financiado parcialmente por el fondo de investigación sanitaria del Ministerio de Sanidad español (proyecto FIS 02/1805) y por el Plan Andaluz de Investigación de la Consejería de Salud de la Junta de Andalucía (proyecto $18 / 03)$.

\section{Bibliografía}

1. Informe del defensor del pueblo sobre las listas de espera [editorial]. Medicina General 2002; 49: 873-4.

2. Ortega Serrano J. Consecución de proyectos ambiciosos de gestión mediante el control organizado del caos. Gestión Hospitalaria 2001; 12: 175-83.

3. Ballesteros Pérez AM, García González AL, Fontcuberta Martínez J, Sánchez Rodríguez F, Pérez Crespo C, Alcázar Manzanera F. La demora en la consulta de atención primaria ¿se puede mejorar?. Aten Primaria $2003 ; 31: 377-81$.

4. Arroyo A, Andreu J, García P, Jover S, Arroyo MA, Fernández A, et al. Análisis de un programa de derivación directa entre atención primaria y especializada en pacientes potencialmente quirúrgicos. Aten Primaria 2001; 28: 381-5.

5. Gérvas J, Palomo L. ¿Alta o excesiva resolución?. Med Clin (Barc) 2002; 119: 315.

6. Granja Romero E, Trinidad San José JC, González Rivero C, Del Río Romero D, Moreno Fernández A, López Soto A. Estudio de las derivaciones a una consulta externa de Medicina Interna: ¿se puede gestionar la lista de espera?. An Med Interna (Madrid) 2004; 21: 72-4.

7. Zambrana García JL, Jiménez-Ojeda B, Marín Patón M, Almazán González S. La consulta única o de alta resolución como una alternativa de eficiencia a las consultas externas hospitalarias tradicionales. Med Clin (Barc) 2002; 118:302-305.

8. Canto Neguilo R. Telemedicina: Informe de evaluación y aplicaciones en Andalucía. Sevilla. Agencia de evaluación de tecnologías sanitarias de Andalucía. $1^{\circ}$ edición. 2000.

9. Rosa F, Zambrana JL, Velasco MJ, Adarraga D, Martos L, Marín M. Análisis de los tiempos de demora en un programa de consulta única. Rev Calidad Asistencial 2002; 17: 378-9.

10. Zambrana JL, Adarraga MD, Rosa F, Velasco MJ, Carmona JA, Montijano AM, et al. Caracterización de procesos asistenciales susceptibles de alta resolución en consultas externas de Medicina Interna. Investigación Clínica 2003: 6 (Supl. 1): 34-5.

11. Montijano Cabrera AM, Zambrana García JL, Amat Vizcaíno M, Velasco Malagón MJ, Adarraga Cansino MD, Rosa Jiménez F, et al. Aplicabilidad y aportaciones al sistema de "Consulta Única" en Cardiología. Rev And Card 2003; 38: 69-76.

12. Bravo Santervás JM, Zambrana García JL, Ruiz Romero JA, Velasco Malagón MJ, Rosa Jiménez F, Amat Vizcaíno M. Impacto sobre el tiempo de espera del modelo de consulta única en Neumología frente a la consulta convencional. Neumosur 2002; 14: 187-92.

13. Puente Gutiérrez JJ, Rodríguez González FJ, Bernal Blanco E, Zambrana García JL. La consulta de alta resolución en aparato digestivo. Rev And Pat Digest 2003; 26: 23s-27s.

14. Ruiz Arranz JL, Ruiz Romero JA, Álvarez JA, Villalón J. La consulta única como instrumento de mejora de la calidad asistencial en cirugía ortopédica y traumatología. Todo Hospital 2001; 111: 375-9.

15. Adarraga MD, Zambrana JL, Rosa F, Velasco MJ, Carmona JA, Sánchez F. Apoyo telemático en consultas ambulatorias hospitalarias: telerradiología y pruebas de laboratorio en un entorno web. Investigación Clínica 2003; 6 (Supl. 1): 13.

16. Puente JJ, Rodríguez FJ, Cano MC, Pelado E, Ramírez A, Zambrana JL. Ansiedad y satisfacción de los pacientes sometidos a gastroscopia en una consulta de alta resolución [abstract]. Rev And Pat Digest 2003; 26: 64 s.

17. Montijano AM, Zambrana JL, Amat M, Moreno M. Grado de satisfacción de pacientes atendidos en un programa de consulta única en cardiología. Rev Calidad Asistencial 2003; 18: 650-4. 